\title{
Caracterização de pessoas submetidas à amputação
}

Recebido em: 12/12/2011

Aceito em: 27/09/2012

\author{
Alexandra Montiel ${ }^{1}$ \\ Mara Ambrosina de Oliveira Vargas ${ }^{2}$ \\ Sandra Maria Cezar Leal ${ }^{3}$
}

Estudo transversal, cujo objetivo foi caracterizar pessoas submetidas à amputação de membros superiores e/ou inferiores em uma rede hospitalar pública de Porto Alegre, no ano de 2009. Os participantes totalizaram 467 pacientes. Os dados foram coletados nos prontuários dos sujeitos do estudo. Utilizou-se o software Epi-Info versão 3.4 .2 no processo de análise. O sexo masculino representou 68,9\%. Destaca-se que, em $95,7 \%$ dos casos, a amputação ocorreu devido a problemas clínicos de saúde, sendo que 50\% deles estavam relacionados ao Diabetes Mellitus. Ainda, 33,8\% e 33,4\% são na altura da coxa e Pododáctilos, respectivamente. Os registros completos qualificam a avaliação da assistência e são indicadores de possíveis intervenções relacionadas à promoção da qualidade do cuidado.

Descritores: Amputação, Amputação Traumática, Assistência à Saúde, Enfermagem.

\section{Characterization of people undergoing amputation}

It is a transversal study aiming to characterize people undergoing amputation of upper and/or lower limbs in a public hospital network in Porto Alegre, 2009. There were 467 participants patients. The data were collected from the records of the subjects of the study. The 3.4.2-version Epi-Info software was used in the analysis process. The male gender represented $68,9 \%$. It is important to emphasize that in $95,7 \%$ of the cases, the amputation occurred due to clinic health problems, and 50\% of them were correlated to Diabetes Mellitus. Yet, 33,8\% and 33,4\% were at the thigh and pododactyls level, respectively. The complete records qualify the care evaluation and are indicators of possible interventions related to the promotion of the care quality. Descriptors: Amputation, Traumatic Amputation, Health Care, Nursing.

\section{Caracterización de personas sometidas a amputación}

Estudio transversal, con el objetivo de caracterizar a las personas sometidas a la amputación de miembros superiores e/o inferiores en una red hospitalaria pública de Porto Alegre, el año 2009. Un total de 467 pacientes participaron del estudio. Los datos fueron recolectados en los prontuarios de los sujetos del estudio. Se utilizó el software Epi-Info versión 3.4.2 en el proceso de análisis. El sexo masculino fue representado por $68,9 \%$. Se destaca que, en $95,7 \%$ de los casos, la amputación ocurrió debido a problemas clínicos de salud, siendo que, en 50\%, estaban relacionados a la Diabetes Mellitus. Y más, 33,8\% y 33,4\% son al nivel del muslo y Pododáctilos, respectivamente. Los registros completos califican la evaluación del cuidado y son indicadores de posibles intervenciones relacionadas a la promoción de la calidad del cuidado.

Descriptores: Amputación. Amputación Traumática. Cuidado de la Salud. Enfermería.

\section{INTRODUÇÃO}

E ste estudo está incluído no projeto de pesquisa "Reabilitação de pacientes submetidos a amputação clínica ou traumática", vinculado ao Curso de Enfermagem da Universidade do Vale do Rio dos Sinos.

É expressivo o número de pessoas que evoluem para amputação de membros superiores e inferiores (MSs e Is) no Brasil(1). Traumas relacionados a acidentes de trânsito e de trabalho, moléstias tropicais, a doenças ateroscleróticas e ao diabetes constituem-se em causas principais da necessidade de amputações ${ }^{(2-3)}$. E, apesar de todos os avanços tecnológicos da saúde, paradoxalmente, a amputação continua sendo uma das opções terapêuticas utilizadas, aumentando o número de deficientes físicos no país.

Amputação é o processo pelo qual se separa do organismo, parcial ou totalmente, mediante cirurgia, um membro ou outra parte do corpo. Ela pode ser considerada um tipo de cirurgia reconstrutora ${ }^{(4)}$. Mas pode-se afirmar que a amputação é uma perda física e psíquica para a pessoa amputada e a compensação dessa perda é um desafio da equipe de saúde ${ }^{(5)}$

O Censo 2010 realizado pelo Instituto Brasileiro de Geografia e Estatística (IBGE) evidenciou que aproximadamente $24 \%$ da população brasileira possui

1 Enfermeira. Graduação em Enfermagem pela Universidade do Vale do Rio dos Sinos - UNISINOS.

2 Enfermeira. Doutora em Filosofia em Enfermagem. Professora do Departamento e Programa de Pós-graduação em Enfermagem da UFSC. Pesquisadora do Grupo de Pesquisa PRÁXIS/UFSC - Trabalho, Cidadania, Saúde e Enfermagem. E-mail: mara@ccs.ufsc.br

3 Enfermeira. Doutora em Enfermagem/UFRGS. Docente da UNISINOS. Membro do GESC/UFRGS. 
alguma deficiência investigada (visual, auditiva, motora, mental/intelectual). A deficiência motora representa 6,95\% da população e, destes, $66,5 \%$ possuem deficiência motora, no mínimo, por alguma dificuldade ${ }^{(6)}$.

No Brasil, estima-se que a incidência de amputações é de 13,9 por 100.000 habitantes/ano. Na literatura mundial, há controvérsias quanto ao número, variando de 2,8 a 43,9 por 100.000 habitantes/ano, sendo mais frequente em pacientes com patologias vasculares ${ }^{(1)}$. Entretanto, além da citada, são várias as etiologias relacionadas à amputação de membros, tais como neuropáticas, traumáticas, tumorais, infecciosas e congênitas ${ }^{(4)}$.

A justificativa do tema escolhido foi baseada na relevância da problemática, sua articulação com a repercussão social e a efetiva abordagem multiprofissional, que atuem de modo interdisciplinar. Destaca-se que faltam publicações referentes ao tema na área da enfermagem, e que é preciso o enfermeiro aprimorar sua inserção e atuação nos cuidados e orientações imprescindíveis para a prevenção de agravos de saúde de origem clínica ou traumática que desencadeiam a necessidade de amputação de MSs e/ou Is e das complicações secundárias ao acometimento de saúde de origem clínica ou traumática. Além disso, a enfermagem desempenha importante papel, tanto no preparo préoperatório, como nos cuidados imediatos após a cirurgia de amputação de MSs e/ou Is, nos cuidados com o coto, visando ao uso da prótese e no preparo para a alta, com as orientações ao paciente e familiares para uma adequada evolução de reabilitação pós-amputação.

O processo de reabilitação se inicia desde a lesão/ doença, podendo se estender por toda a vida. Dessa forma, envolvendo o tratamento no hospital, na comunidade e no ambiente da pessoa com deficiência, a reabilitação deve proporcionar apoio psicológico e educação em saúde buscando solucionar preocupações individuais a respeito da nova condição(7).

Nessa perspectiva, a questão norteadora é "quem são as pessoas submetidas a uma amputação de MSs e/ou Is"?

O objetivo do estudo é caracterizar as pessoas submetidas à amputação de MSs e/ou Is em uma rede hospitalar pública de Porto Alegre, no ano de 2009, quanto ao sexo, faixa etária, raça/cor, ocupação profissional, procedência, causa do agravo e doenças associadas.

\section{MÉTODO}

Estudo transversal realizado em uma rede hospitalar pública de Porto Alegre/RS, da qual foram incluídos dois hospitais: um deles é referência no atendimento de emergências traumáticas; o outro é um Hospital Geral no qual funciona um serviço de referência no atendimento a pessoas com doença vascular.

Os participantes foram todos os sujeitos com idade a partir de 18 anos submetidos à amputação de membros superiores e/ou inferiores, nos hospitais em estudo, no ano de 2009. A coleta dos dados realizou-se nos prontuários desses
Tabela 1- Distribuição quanto ao tipo de causa da amputação, o sexo e a faixa etária dos pacientes submetidos à amputação em uma rede hospitalar pública de Porto Alegre/ RS no ano de 2009.

\begin{tabular}{|c|c|c|c|c|}
\hline Variáveis & \multicolumn{2}{|c|}{$\begin{array}{c}\text { Amputação } \\
\text { Clínica } \\
N=447\end{array}$} & \multicolumn{2}{|c|}{$\begin{array}{c}\text { Amputação } \\
\text { Traumática } \\
N=20\end{array}$} \\
\hline Sexo & $\mathbf{F}$ & $\%$ & $\mathbf{F}$ & $\%$ \\
\hline Masculino & 308 & 68,90 & 14 & 70,00 \\
\hline Feminino & 139 & 31,10 & 06 & 30,00 \\
\hline Total & 447 & 100,00 & 20 & 100,00 \\
\hline Faixa etária & $\mathbf{F}$ & $\%$ & $\mathbf{F}$ & $\%$ \\
\hline$<20$ anos & 02 & 0,4 & 02 & 10,0 \\
\hline 21 a 40 anos & 17 & 3,8 & 13 & 65,0 \\
\hline 41 a 60 anos & 176 & 39,4 & 04 & 20,0 \\
\hline 61 a 80 anos & 218 & 48,8 & 01 & 5,0 \\
\hline$>$ de 80 anos & 34 & 7,6 & 0 & 0,0 \\
\hline Total & 447 & 100,00 & 20 & 100,00 \\
\hline Raça/cor & $\mathbf{F}$ & $\%$ & $\mathbf{F}$ & $\%$ \\
\hline Branca & 385 & 86,1 & 16 & 80,0 \\
\hline Negra & 60 & 13,4 & 04 & 20,0 \\
\hline Sem o registro & 02 & 0,4 & 0 & 0,0 \\
\hline Total & 447 & 99,9 & 20 & 100,00 \\
\hline
\end{tabular}

Fonte: Montiel, 2011.

pacientes. Para a análise utilizou-se o software Epi-Info versão 3.4.2, com a categorização dos eventos, cruzamento de variáveis, descrição de índices frequenciais absolutos e relativos.

Seguiram-se as normas preconizadas na Resolução 196/1996 do Conselho Nacional de Saúde. O projeto de pesquisa foi aprovado pelo Comitê de Ética e Pesquisa da instituição, por meio do parecer n 11-059.

Tabela 2 - Distribuição quanto ao tipo de causa da amputação e a procedência dos pacientes submetidos a amputação em um grupo hospitalar público de Porto Alegre/RS no ano de 2009.

\begin{tabular}{|c|c|c|c|c|}
\hline \multirow[t]{2}{*}{ Procedência } & \multicolumn{2}{|c|}{$\begin{array}{c}\text { Amputação } \\
\text { Clínica } \\
\text { N= } 447\end{array}$} & \multicolumn{2}{|c|}{$\begin{array}{l}\text { Amputação } \\
\text { Traumática } \\
\qquad N=20\end{array}$} \\
\hline & $\mathbf{F}$ & $\%$ & $\mathbf{F}$ & $\%$ \\
\hline Porto Alegre & 207 & 46,3 & 06 & 30,0 \\
\hline Região Metropolitana & 179 & 40,0 & 10 & 50,0 \\
\hline Litoral & 21 & 4,7 & 01 & 5,0 \\
\hline Interior do Estado & 37 & 8,3 & 03 & 15,0 \\
\hline Serra & 03 & 0,7 & 0 & 0,0 \\
\hline Total & 447 & 100,0 & 20 & 100,0 \\
\hline
\end{tabular}

Fonte: Montiel, 2011. 
Tabela 3 - Distribuição quanto às doenças e causas associadas, dos pacientes submetidos a amputação em um grupo hospitalar público de Porto Alegre/RS no ano de 2009.

\begin{tabular}{|l|cc|cc|} 
Doença associada & \multicolumn{2}{|c|}{$\begin{array}{c}\text { Amputação } \\
\text { Clínica } \\
\text { N=447 }\end{array}$} & \multicolumn{2}{c|}{$\begin{array}{c}\text { Amputação } \\
\text { Traumática } \\
\text { N= 20 }\end{array}$} \\
\hline Diabetes & $\mathbf{F}$ & $\%$ & $\mathbf{F}$ & $\%$ \\
\hline Doenças vasculares & 223 & 50,0 & 0 & 0,0 \\
Hipertensão arterial & 114 & 25,5 & 0 & 0,0 \\
\hline Infecção & 45 & 10,1 & 0 & 0,0 \\
Acidente vascular cerebral & 16 & 3,6 & 5 & 25,0 \\
Outros & 10 & 2,2 & 0 & 0,0 \\
Trauma & 14 & 3,1 & 1 & 5,0 \\
Câncer & 2 & 0,4 & 0 & 0,0 \\
\hline Prontuário sem informação & 2 & 0,4 & 0 & 0,0 \\
\hline Total & 21 & 4,7 & 14 & 70,0 \\
\hline
\end{tabular}

Fonte: Montiel, 2011.

\section{RESULTADOS}

No período do estudo, de janeiro a dezembro de 2009, 467 pacientes foram submetidos a amputação de MSs e/ ou Is na rede hospitalar em estudo. Para a análise, os casos foram classificados em dois tipos de causas de amputação: clínicas e traumáticas. Ou seja, as primeiras são causadas por patologia; as traumáticas são as que ocorreram por consequência de agravo por fatores externos (acidentes, quedas, queimaduras, entre outros) e podem ter ocorrido no momento do acidente ou resultante do trauma.

Destaca-se que o tipo de amputação clínica foi o mais frequente e representou $95,7 \%$ dos casos. A seguir, na Tabela 1, apresenta-se a distribuição dos sujeitos amputados, quanto ao sexo, faixa etária e raça/cor.

Na distribuição quanto ao tipo de causa da amputação e ao grupo profissional dos pacientes submetidos à amputação, detectado que na amputação clínica predominaram: $21,5 \%$ eram do lar e $1,8 \%$ eram aposentados/pensionistas; entre os trabalhadores, $12,8 \%$ em obras e

"estudos sobre o perfil de pessoas amputadas também identificaram os homens como o grupo mais atingido(8-10), possivelmente devido ao fato de que as pessoas do sexo masculino estão mais expostas"
Quanto às doenças associadas descritas na Tabela 3 , sinaliza-se que na maioria dos prontuários havia associação de várias patologias. Logo, optou-se por organizar as informações, agrupando os dados, priorizando destacar a principal causa. Nas doenças vasculares, foi agrupada a doença vascular periférica e a doença obstrutiva arterial periférica.

As infecções, principalmente a gangrena, estavam associadas a $25 \%$ das causas das amputações traumáticas. Entretanto, não foi possível relacionar doenças e causas associadas em $70 \%$ dos casos, porque não havia o registro da informação nos prontuários.

Ainda, a distribuição quanto à região do corpo amputada, na amputação clínica concentraram, dentre os 447 pacientes: 151 da coxa, 149 dos pododáctilos, 69 da perna, 44 do antepé, 12 do pé, nove dos quirodáctilos, quatro do joelho, dois do braço, dois da supracondiliana e dois do tornozelo. Evidenciados três prontuários sem o respectivo registro. Já na amputação traumática, os resultados sinalizam, dentre os 20 pacientes: quatro amputação da coxa, dois da perna, um do pé, nove dos quirodáctilos, um do braço, um da supracondiliana, um na mão e um no punho.

\section{DISCUSSÃO}

Nas informações referentes à caracterização das pessoas submetidas a uma amputação de MSs e/ou Is na rede hospitalar em estudo, o sexo masculino foi o mais frequente e representou $68,90 \%$ nas amputações clínicas e $70 \%$ nas traumáticas. Resultados de outros estudos sobre o perfil de pessoas amputadas também identificaram os homens como o grupo mais atingido(8-10), possivelmente devido ao fato de que as pessoas do sexo masculino estão mais expostas a traumas físicos decorrentes de acidentes e/ou serem as mais acometidas por amputações causadas por complicações vasculares ${ }^{(1)}$.

Neste estudo, a faixa etária mais atingida não foi a mesma para os indivíduos submetidos à amputação clínica e traumática. Nas amputações clínicas, a faixa etária mais frequente foi de 61 a 80 anos. Em pesquisa realizada no Hospital Regional de Cascavel/PR, estudiosos sobre o tema também encontraram maior índice de amputação por causa clínica e argumentam que a idade avançada, associada a algum comprometimento da habilidade física e a maior dependência, pode justificar tal achado ${ }^{(1-11)}$

Na faixa etária acima de 60 anos, as amputações ocorrem principalmente nos pacientes diabéticos, e tendem a aumentar a incidência com o envelhecimento ${ }^{(1)}$. Já nas amputações traumáticas, a faixa etária de 21 a 40 anos 
representou $65 \%$, dado esse que corrobora com estudo realizado em Guarapuava/PR, com indivíduos submetidos a amputação de membros Is, cujos resultados apontaram que $41,18 \%$ eram uma população de adultos jovens ${ }^{(12)}$.

Resultado de outro estudo realizado na Universidade Estadual de Maringá sobre amputações e desbridamentos de membros Is também identificou que a maioria era uma população jovem e do sexo masculino(10). Esses acidentes ocorrem na época mais produtiva da vida, causando sequelas irreversíveis ${ }^{(13-14)}$. Nessa perspectiva, vários pesquisadores enfatizam que as amputações traumáticas acometem mais os indivíduos com menos de 50 anos, devido à maior exposição no trabalho e no trânsito ${ }^{(9,11,14-15)}$.

Neste estudo, em especial os sujeitos submetidos à amputação clínica exerciam, principalmente, a profissão do lar, ou seja, não estavam inseridos formalmente no mercado de trabalho, enquanto que nas causas traumáticas a profissão que se destacou foi a autônoma. Considerase que esses resultados representam as circunstâncias nas quais ocorrem cada tipo de amputação. Assim, as motivadas por eventos clínicos estão associadas a doenças crônicas, que já haviam causado algum tipo de incapacidade para o trabalho, ou a pessoa estava aposentada por idade ou tempo de serviço. Porém, nos eventos traumáticos, além da faixa etária estar relacionada à idade produtiva (65\% entre 20 e 40 anos de idade), em muitas situações o acidente ocorreu no próprio local de trabalho, no caso da construção civil, que representou $10 \%$ dos sujeitos com esse tipo de amputação. Além disso, os acidentes de trajeto, configurados como acidente de trabalho, também representaram importantes índices de ocorrência, ainda que em $30 \%$ dos prontuários dos pacientes submetidos à amputação traumática não constava a informação relacionada ao tipo de ocupação. Estudo ${ }^{(16)}$ corrobora que os acidentes de trabalho estão entre as principais causas de traumas, envolvendo fraturas expostas graves, com risco de amputação, em especial entre os motoboys.

Em outra pesquisa sobre amputação de extremidades, identificou-se a falta de registros relacionados à profissão em $48,2 \%$ dos prontuários ${ }^{(17)}$. Ressalta-se que as informações incompletas nos prontuários interferem na comunicação entre a equipe multiprofissional. Além disso, desqualificam a avaliação da assistência à saúde, quando o critério é o uso de base de dados administrativos, que têm os prontuários como fonte ${ }^{(18)}$.

Quanto às causas associadas à amputação de extremidades, o diabetes é a principal, seguida pelas doenças vasculares. Vale dizer que o diabetes é um fator que aumenta o risco de insuficiência vascular, estreitando a relação com a indicação de amputação de extremidades.
Os resultados deste estudo vão ao encontro de outras pesquisas que indicam essas doenças como os principais motivos de amputação por causa clínica tanto para homens como para mulheres ${ }^{(1 ; 10)}$. Já nas amputações traumáticas, as causas associadas são as infecções (25\%) e em $75 \%$ dos prontuários não há o registro da informação. Resultados de estudos acerca do tema destacam que essas amputações estão relacionadas à maior exposição no trabalho e no trânsito, entre a população de adultos jovens ${ }^{(19)}$.

Em relação ao local do corpo, as amputações na região da coxa representaram 33,8\% nas decorrentes de causas clínicas e dos quirodáctilos, $45 \%$ nas causadas por evento traumático. Em outro estudo sobre caracterização de pacientes com membros amputados é identificado que, nas causas vasculares, o maior número de amputações se dá ao nível da coxa e que, segundo os pesquisadores, é devido ao comprometimento da circulação arterial fêmoro-poplítea. Já nas causas traumáticas, o maior número ocorre ao nível dos quirodáctilos e está relacionado a acidentes domésticos e de trabalho(8).

Os hospitais em estudo situamse em Porto Alegre. Um deles é referência no atendimento vascular e o outro no atendimento ao trauma. A maioria dos sujeitos submetidos a uma amputação residia no município de Porto Alegre (46,3\%), seguido da Região Metropolitana $(40,0)$ e de outras regiões $(13,7 \%)$. Porém, pôde-se considerar que $53,7 \%$ eram oriundos de cidades diferentes da localização das instituições. Isso pode indicar o acesso restrito desse tipo de assistência à saúde para os usuários do Sistema Único de Saúde que não são moradores da cidade de Porto Alegre, pois precisam deslocar-se para ser atendidos em um serviço especializado, situações agravadas quando existe o risco de morte, como nos casos das urgências vasculares, dos acidentes graves de trânsito e de trabalho.

\section{CONCLUSÃO}

$\mathrm{Na}$ caracterização da população em estudo, identificou-se que os homens foram os mais submetidos à amputação, tanto clínica como traumática, provavelmente porque estão mais expostos a acidentes e a doenças vasculares. A faixa etária mais frequente nas amputações clínicas foi a de 61 a 80 anos, principalmente em pacientes com diabetes e doenças vasculares, enquanto nas causas traumáticas a faixa etária 21 a 40 anos representou $65 \%$, envolvendo maior exposição de risco no trabalho e no trânsito. Porém, nas amputações clínicas, a maioria dos acometidos desenvolvem suas atividades ocupacionais no domicílio e estão excluídos do mercado de trabalho. Nas amputações clínicas, o diabetes foi a principal doença associada, seguido 
pelas doenças vasculares, enquanto que na traumática as infecções foram as que prevaleceram.

Considera-se que apesar de poucas pesquisas sobre o tema na área da enfermagem, este estudo poderá contribuir para o planejamento do cuidado, com abordagem específica a essa população. Além disso, ações de Educação e Saúde voltadas à prevenção das complicações do diabetes podem favorecer a redução das amputações clínicas. Nessa perspectiva, a prevenção de acidentes de trabalho e de trânsito também pode auxiliar a diminuir as amputações decorrentes desses agravos.
Destaca-se que alguns resultados ficaram comprometidos pela falta de registros ou por registros incompletos nos prontuários, principalmente nos dados referentes à ocupação, procedência, doenças associadas e região do corpo (não foi possível identificar se as amputações supracondilianas estavam relacionadas ao úmero ou ao fêmur).

Ressalta-se que a enfermagem desempenha importante papel nos cuidados prestados ao paciente submetido a uma amputação. Assim, os registros completos qualificam a avaliação da assistência e são indicadores de possíveis intervenções relacionadas à promoção da qualidade do cuidado.

\section{Referências}

1. Carvalho FS. Prevalência de amputação em membros inferiores de causa vascular: análise de prontuários. Arq Ciênc Saúde Unipar. 2005;9(1):23-30. 2. Chan ACRV. Incidência de amputação em membros inferiores associada a diabetes mellitus [Internet]. [citado em 2011 Out 10]. Disponível em: http:// redalyc.uaemex.mx/src/inicio/ArtPdfRed.jsp?iCve=84212107007.

3. Chini GCO. Amputação sob uma perspectiva fenomenológica [dissertação]. Ribeirão Preto: Escola de Enfermagem de Ribeirão Preto da Universidade de São Paulo; 2005. 4. Smeltzer SC, Bare BG. Brunner \& Suddarth: tratado de enfermagem médicocirúrgica. $11^{\mathrm{a}}$ ed. Rio de Janeiro: Guanabara Koogan; 2009.

5. O'Keeffe B. Prosthetic rehabilitation of the upper limb amputee [Internet]. [citado em 2012 Mai 17]. Disponível em: http://www.ijps.org/article.

asp?issn=0970-0358; year=2011; volume=44;issue=2; ;page=246;epage=252; aula $\mathrm{st}=\mathrm{O} \% 27 \mathrm{Keeffe}$

6. Instituto Brasileiro de Geografia e Estatística. Censo 2010 [Internet]. [citado em 2012 Jun 23]. Disponível em: http://www.ibge.gov.br/home/estatistica/ populacao/censo2010/resultados_preliminares_amostra/default_resultados_ preliminares_amostra.shtm

7. Perkins ZB, De'Ath HD, Sharp G, Tai NRM. Factors affecting outcome after traumatic limb amputation [Internet]. \{citado em 2012 Jul 22]. Disponível em: http://onlinelibrary.wiley.com/doi/10.1002/bjs.7766/pdf

8. Agne JE, Cassol CM, Bataglion D, Ferreira FV. Identificação das causas de amputações de membros no hospital universitário de Santa Maria. Saúde. 2004;30(1/2):84-9.

9. Cassefo V, Nacaratto DC, Chamlian TR. Perfil epidemiológico dos pacientes amputados do Lar Escola São Francisco: estudo comparativo de 3 períodos diferentes. Acta Fisiátrica. 2003;10(2):67-71.

10. Seidel AC. Epistemologia sobre amputações e desbridamentos de membros inferiores realizados no Hospital Universitário de Maringá. J Vascular Bras. 2008;7(4):308-15.

11. Carvalho JA. Amputações de membros inferiores em busca da plena

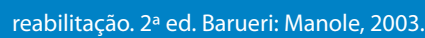

12. Teixeira G, Novak VC. Nível de independência física dos amputados de membros inferiores do município de Guarapuava - PR. In: XII Encontro Latino Americano de Iniciação Científica; IX Encontro Latino Americano de Pósgraduação; III Encontro Latino Americano de Iniciação Científica Júnior; 16-17 out. 2009; São José dos Campos, 2009 [Internet]. [citado em 2011 Out 22]. Disponível em: http://www.inicepg.univap.br/cd/INIC_2009/anais/arquivos/ RE_0643_1065_01.pdf.

13. Tolotti VC, Silva LAA. Caracterização das vítimas de trauma atendidas em emergência hospitalar no norte do estado do Rio Grande do Sul. Contexto Saúde. 2004;3(7):191-8.

14. Camacho-Conchucos HT. Patients amputated by work accidents: characteristics and years accumulated of potential productive life lost. An Fac Med. 2010;71(4):271-5.

15. Brito DD, Isernhagen FC, Depiere TZ. Tratamento fisioterapêutico ambulatorial em paciente submetido à amputação transfemoral unilateral por acidente motociclístico: estudo de caso [Internet]. [citado em 2011 Mai 06]. Disponível em: http://revistas.unipar.br/saude/article/viewFile/193/167

16. Santos AMR. Perfil das vítimas de trauma por acidente de moto atendidas em um serviço público de emergência. Cad Saúde Pública. 2008;24(8):927-38.

17. Tavares DMS. Perfil de clientes submetidos a amputações relacionadas ao diabetes mellitus. Rev Bras Enferm. 2009;62(6):825-30.

18. Vasconcellos MM, Gribel EB, Moraes IHS. Registros em saúde: avaliação da qualidade do prontuário do paciente na atenção básica, Rio de Janeiro, Brasil. Cad Saúde Pública. 2008;24(1):s173-s82.

19. Umburanas RC. Amputação de membro inferior: perfil dos pacientes do serviço de reabilitação física da unicentro - projeto órtese e prótese. In: XII Encontro Latino Americano de Iniciação Científica; IX Encontro Latino Americano de Pós-graduação; III Encontro Latino Americano de Iniciação Científica Júnior; 16-17 out. 2009; São José dos Campos; 2009 [Internet]. [citado em 2011 Mai 04]. Disponível em: http://www.inicepg.univap.br/cd/INIC_2009/anais/ arquivos/0643_0982_01.pdf. 